\title{
Comparative analysis of current and magnetic multipole graphical models
}

\author{
Shi-Qin Jiang, Lu Bing, Jia-Ming Dong, Ming Chi, Wei-Yuan Wang, Lei Zhang
}

School of Electronics and Information Engineering, Tongji University, Shanghai, China.

Email: sqjiang@tongji.edu.cn

Received 28 July 2009; revised 1 September 2009; accepted 2 September 2009.

\begin{abstract}
In recent year, a multipole graphical model, which is constructed by using individual MCG measurements based on the equivalent current dipole (ECD) or equivalent magnetic dipole (EMD) source model, has been developed with the aim of instead of the volume conductor model in the inverse solution of cardiac source estimation. In this paper, two graphical models known as the double magnetic dipole source model (DMD) and the dual current dipole source model (DCD) are introduced. The simulation results and the comparison of two evaluation criteria, i.e. average GOF (Goodness of Fit) and average RMSE (Root Mean Square Error), indicated that both multipole graphical models can provide a good representation of dynamic magnetic field from the noninvasively detected MCGrecordings, even when the heart is of the dilation. The time-averaged sources localization error and the RMSE for both models are demonstrated, and the characteristic of two multipole models is discussed.
\end{abstract}

Keywords: Biomagnetics; Inverse Problems; Dipole Source Localization; Modeling; Magnetocardiography

\section{INTRODUCTION}

In order to investigate cardiac electrical activity, the issue of reconstructing noninvasively the electrical or magnetic sources from detected MCG signals has received much attention since 1970'.

Due to the effect of human torso, the volume conductor model, i.e., heart-torso model, such as 3D finite element model (FEM), boundary element model (BEM), and ventricular propagated excitation model were developed $[1,2,3]$. Front two models are created with magnetic resonance imaging (MRI) data and conductivity values are assigned to each region. It is demonstrated that the use of torso models has brought significant improvements in results of dipole source localization.

In recent years, for both research and clinical applications, we developed a graphical model (GM) to describe the active magnetic field between detected MCG data and cardiac electrical sources [4]. The aim is to provide a simple model which can describe the varying magnetic field and conductivity properties of tissue. Furthermore, two multipole source models known as the double magnetic dipole source model (DMD) and the dual current dipole source model (DCD) are investigated. In Figure 1, there are three graphical models which are constructed based on different source models [5,6,7].

The graphical model consists of a set of magnetic field maps (MFM), which is constructed by using individual MCG measurements based on the equivalent current dipole (ECD) or equivalent magnetic dipole (EMD) source model. Each graphical model includes 25 magnetic field maps with a time interval of $4 \mathrm{~ms}$ during 100 ms in ST-T segment. Each map of the graphical model corresponds on a set of model parameters, by which the space time pattern of the magnetic field over the body surface can be obtained with high-resolution. The procedure of model constructing is illustrated in Figure 2. It is implemented with three steps: initial values determination, source estimation, and GM construction in terms of optimized source parameters, which are estimated by applying the Levenberg-Marquart (LM) or the NelderMead (NM) algorithm.

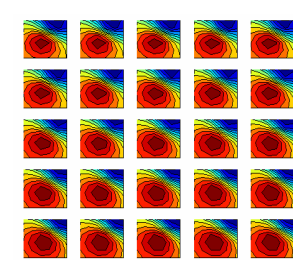

(a) GM of SCD

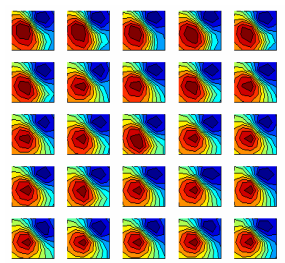

(c) GM of DCD

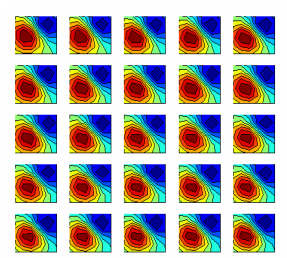

(b) GM of DMD

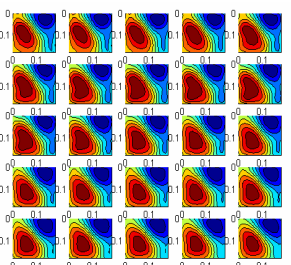

(d) Detected MCG data
Figure 1. Three graphical models and detected MCG data. 


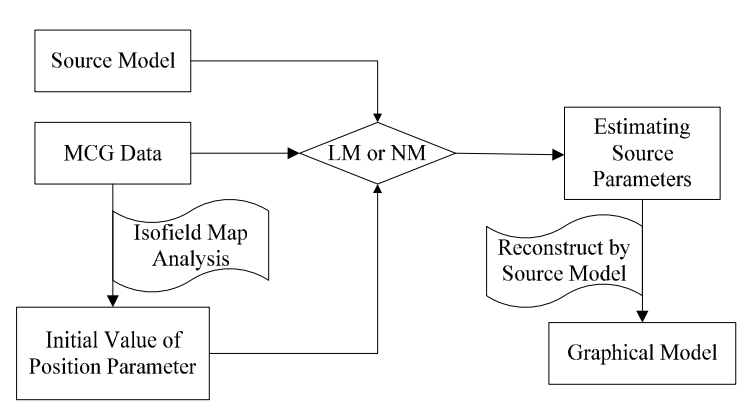

Figure 2. Schematic diagram of constructing a graphical model.

Two multipole source models mentioned above, i.e., DMD and DCD, have different characteristic from current dipole and magnetic dipole. The double magnetic dipole is similar as a single current dipole (SCD), which is more suitable for experimental implementation. The dual current dipole is originally used to research the method of constructing a multipole source model. In this paper, sources localization results of both source models are demonstrated, and the characteristics of two different multipole graphical models are discussed.

\section{METHODS}

\subsection{Double Magnetic Dipole Source Model}

A simplified double magnetic dipole source model has developed with a pair of magnetic dipole as shown in Figure 3. The necessary conditions of the model are: one of magnetic moments should be positive, and another should be negative. Two magnetic dipoles under the surfing and the sinking of the detected magnetic field over the body surface at the same time. In other words, the magnetic moments are simplified as all vertical to measuring plane and opposite in direction. Therefore, the simplified double magnetic dipole is similar to an equivalent current dipole. However, the model has more parameters than that of SCD model, thus, it has more degree of freedom.

The magnetic field $\mathrm{B}_{\mathrm{z}}$ detected along the $\mathrm{Z}$-axis, which generated by a pair of magnetic dipole, is defined as:

$$
B\left(x_{j}, y_{j}\right)=\sum_{i=1}^{2} \frac{m_{i} \mu_{0}\left(2 z_{0 i}{ }^{2}-\left(x_{j}-x_{0 i}\right)^{2}-\left(y_{j}-y_{0 i}\right)^{2}\right)}{4 \pi\left(\left(x_{j}-x_{0 i}\right)^{2}+\left(y_{j}-y_{0 i}\right)^{2}+z_{0 i}{ }^{2}\right)^{5 / 2}}
$$

where $\left(x_{0 i}, y_{0 i}, z_{0 i}\right)(i=1,2)$ is the dipole position and $m_{t}(i=1,2)$ is the magnetic moment of two magnetic dipoles, respectively.

\subsection{Dual Current Dipole Source Model}

The dual current dipole source model was developed as the simplest multipole current source model. A magnetic field zero line (MFZL) method was proposed for deter-

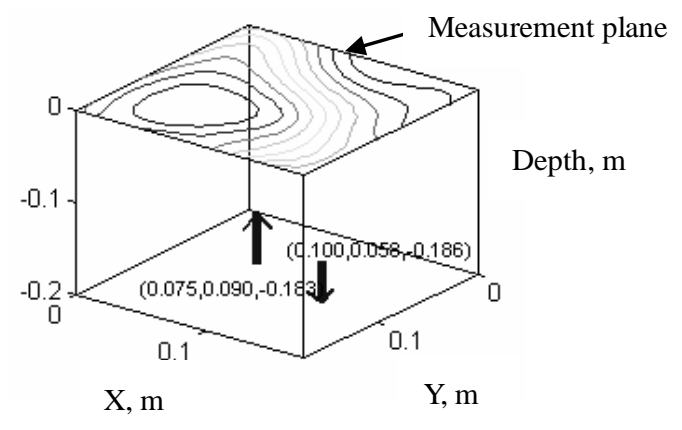

Figure 3. Sketch of a pair of magnetic dipole under the measurement plane.

mining the number of dipoles and the initial values in multiple source inverse solution. In general, the slope of the magnetic field zero line (MFZL) of magnetic field maps is mutative, which has been shown in Figure 4(a). There are 25 varying MFZLs in the ST-T segment obtained from the detected magnetocardiograms in Figure 1(d). As shown in Figure 4(b), the MFZL can be roughly divided into several linear subsections, here are two subsections, according to its slope transition. Thus, we assume that there exist two current dipoles. Every one locates at the middle of a MFZL subsection. The strength of each current dipole depends on the length of the corresponding MFZL subsection [8]. Obviously, one current dipole is dominant; another is an accessorial equivalent dipole. Based on the above a priori assumptions, an advisable method of constructing a multipole source model is to locate a current dipole on a MFZL subsection. The method can be used to determine the initial values of dipoles in inverse solution. The multiple current dipoles source model is defined as:

$$
B\left(x_{j}, y_{j}\right)=\sum_{i=1}^{n} \frac{\left(y_{j}-y_{i}\right) \cdot \mu_{0} Q_{i x}-\left(x_{j}-x_{i}\right) \cdot \mu_{0} Q_{i y}}{4 \pi\left(\left(x_{j}-x_{i}\right)^{2}+\left(y_{j}-y_{i}\right)^{2}+z_{i}^{2}\right)^{3 / 2}}
$$

where (xi, yi, zi) is the position of the current dipole, Qx and Qy are the $\mathrm{x}$ and $\mathrm{y}$ components of current moments of the dipole, respectively. $\mathrm{N}$ is the number of dipoles.

The simulation results of the dual current dipole are presented as followings. It demonstrated that the accuracy of the graphical model was improved by means of the multipole current source model.

\section{SIMULATION RESULTS}

\subsection{Accuracy of Graphical Models}

It is necessary that the graphical model is of high accuracy for describing the detected MCG contour maps. The accuracy of graphical models depends on the accuracy of sources estimation. Thus, the source model and the algorithm play an important role in inverse solution. In terms of two evaluation criteria, i.e. GOF (Goodness of Fit) and RMSE (Root Mean Square Error), a comparison 
performance among three graphical models is given in Figure 5 and Figure 6, respectively. Two evaluation criteria are defined as followings:

$$
\begin{gathered}
G O F=\sqrt{1-\frac{\sum_{i=1}^{N}\left(B_{z i}-B_{s i}\right)^{2}}{\sum_{i=1}^{N} B_{z i}{ }^{2}}} \\
R M S E=\sqrt{\frac{1}{N} \sum_{i=1}^{N}\left(B_{z i}-B_{s i}\right)^{2}}
\end{gathered}
$$

where $B_{z}$ is the detected magnetic field, $B_{s}$ is the calculated magnetic field, and $\mathrm{N}$ is the number of measuring points. Figure 5 and Figure 6 demonstrated that two graphical models, i.e. DCD and DMD, all have higher GOF (more than 0.97) and lower RMSE (about $30 \mathrm{pT}$ ), compared with that of the SCD model.

\subsection{Source Localization}

The accuracy of equivalent dipole source localization is tested based on the double magnetic dipole and the dual current dipole graphical models by using the MCG measurements as shown in Figure 1(d). The inverse prob-

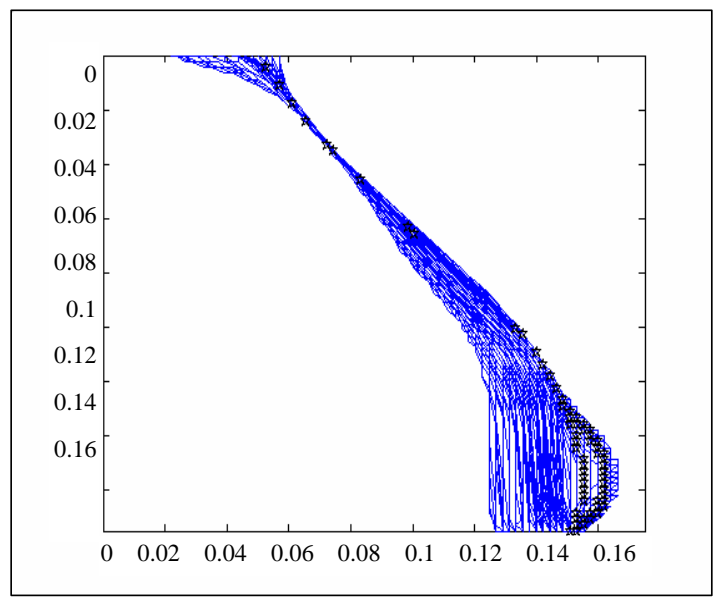

Figure 4. Schematic diagram of magnetic field zero line.

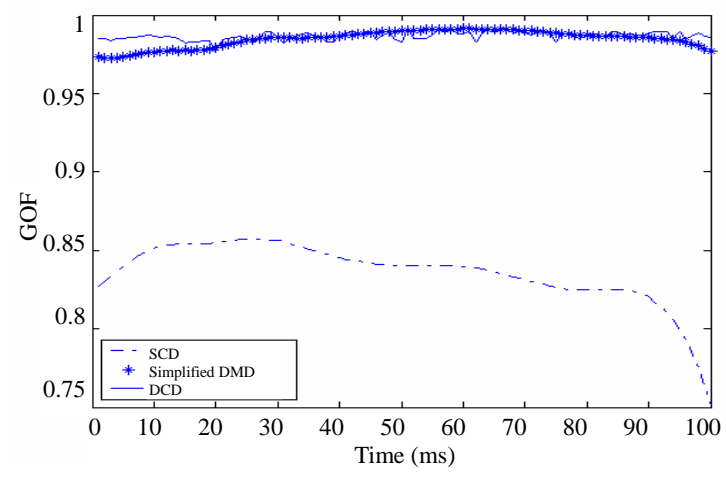

Figure 5. GOF curves of three GMs. lems are calculated with two non-linear local optimization algorithms, LM and NM algorithms, respectively. Two noise levels are considered in simulation, i.e. no noise case and 20dB signals-noise-ratio (SNR), respectively. The initial values are determined by means of a parameters calculation procedure. In simulation two cases were included: use of the calculated initial values and use of the calculated initial value adding a random number whose magnitude is $10 \%$ of the calculated initial values.

The time-averaged localization error and the time-averaged RMSE in 100ms ST-T segment with the DMD and DCD models are shown in Tables 1-2 and Tables 3-4, respectively. In Table 1, the $\mathrm{P}$ expresses the positive magnetic dipole, and the $\mathrm{N}$ is the negative magnetic dipole. In Table 3, D and A express the dominant current dipole and the accessorial equivalent dipole, respectively. We can see that in most cases, the NM algorithm performs better than the LM algorithm regarding both of the averaged localization error and the averaged RMSE. By using NM algorithms, the sources localization results of the DCD model are $0.99 \mathrm{~mm}$ for the dominant dipole and $1.23 \mathrm{~mm}$ for the associate equivalent dipole when calculated initial values are
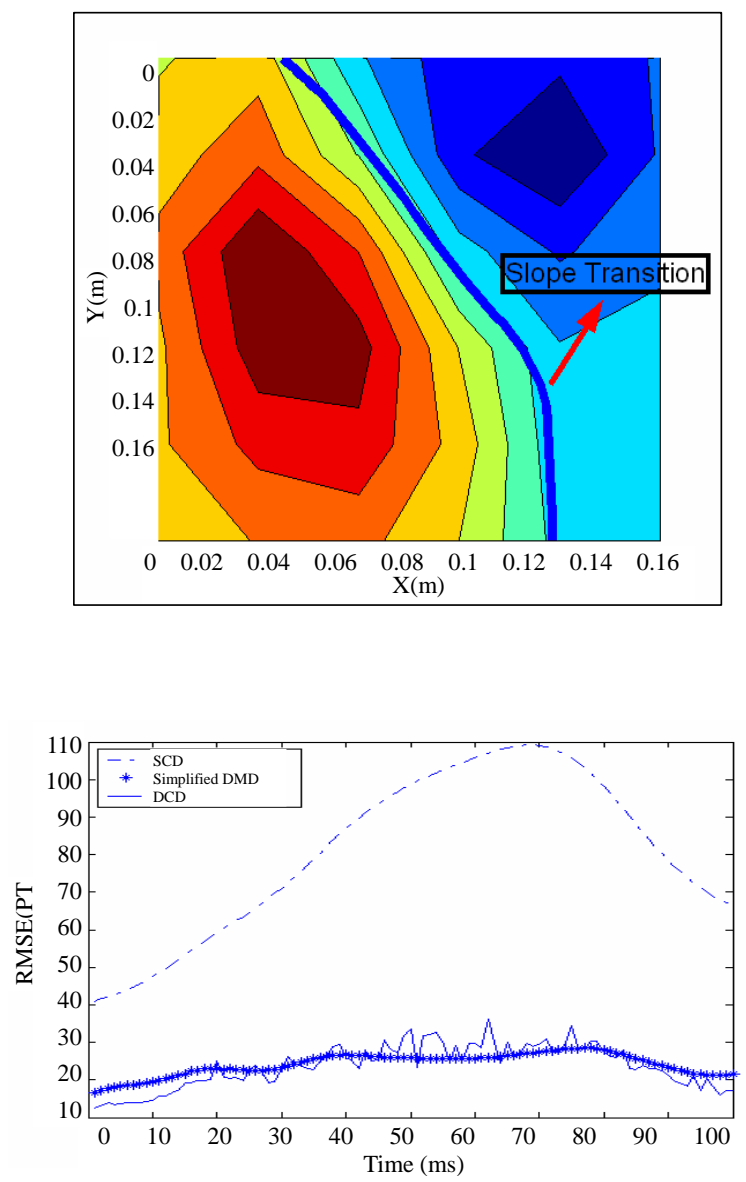

Figure 6. RMSE curves of three GMs. 
Table 1. Averaged localization error of DMD model (mm).

\begin{tabular}{lccccc}
\hline & $\begin{array}{c}\text { Initial } \\
\text { values }\end{array}$ & \multicolumn{2}{c}{ Calculated Initial Values } & \multicolumn{2}{c}{$10 \%$ Random } \\
\cline { 2 - 6 } $\begin{array}{l}\text { Data } \\
\text { noises }\end{array}$ & & $\boldsymbol{L M}$ & $\boldsymbol{N M}$ & $\boldsymbol{L} \boldsymbol{M}$ & $\boldsymbol{N} \boldsymbol{M}$ \\
\hline No & $\mathrm{P}$ & 0.0 & 0.0 & 0.34 & 0.41 \\
noise & $\mathrm{N}$ & 0.0 & 0.0 & 0.69 & 1.06 \\
$20 \mathrm{db}$ & $\mathrm{P}$ & 1.34 & 1.86 & 1.39 & 1.40 \\
noise & $\mathrm{N}$ & 3.69 & 4.84 & 3.74 & 3.72 \\
\hline
\end{tabular}

Table 2. Averaged RMSE of DMD model (pt).

\begin{tabular}{ccccc}
\hline $\begin{array}{l}\text { Initial } \\
\text { values } \\
\text { noises }\end{array}$ & \multicolumn{2}{c}{ Calculated Initial Values } & \multicolumn{2}{c}{ 10\% Random } \\
\cline { 2 - 5 } No noise & 0.0 & 0.0 & 189 & $\boldsymbol{N M}$ \\
\hline 20db noise & 232 & 187 & 255 & 196 \\
\hline
\end{tabular}

Table 3. Averaged localization error of DCD model (mm).

\begin{tabular}{lccccc}
\hline \multirow{2}{*}{$\begin{array}{l}\text { Data } \\
\text { noises }\end{array}$} & $\begin{array}{l}\text { Initial } \\
\text { vaues }\end{array}$ & Calculated Initial Values & \multicolumn{2}{l}{ 10\% Random } \\
\cline { 2 - 6 } & & $\boldsymbol{L M}$ & $\boldsymbol{N M}$ & $\boldsymbol{L} \boldsymbol{N}$ & $\boldsymbol{N M}$ \\
\hline No & $\mathrm{D}$ & 0.0 & 0.0 & 1.14 & 0.89 \\
noise & $\mathrm{A}$ & 0.0 & 0.00 & 1.43 & 0.96 \\
$20 \mathrm{db}$ & $\mathrm{D}$ & 1.20 & 0.99 & 1.71 & 0.99 \\
noise & $\mathrm{A}$ & 1.79 & 1.22 & 1.88 & 1.23 \\
\hline
\end{tabular}

Table 4. Averaged RMSE of DCD model (PT).

\begin{tabular}{ccccc} 
Initial & \multicolumn{2}{l}{ Calculated Initial Values } & \multicolumn{2}{l}{$10 \%$ Random } \\
\cline { 2 - 5 } vata & $\boldsymbol{L M}$ & $\boldsymbol{N M}$ & $\boldsymbol{L} \boldsymbol{N}$ & $\boldsymbol{N M}$ \\
noises & & & & \\
\hline No noise & 0.0 & 0.0 & 211 & 112 \\
20db noise & 228 & 137 & 236 & 155 \\
\hline
\end{tabular}

used. In despite of the localization results of the DMD model have been improved, but they are still not as good as that of the DCD model.

Furthermore, Figure 7 to Figure 8 show the timevarying position and orientation curves of two magnetic dipoles in the $100 \mathrm{~ms}$ ST-T segment. The positive magnetic dipole, i.e. the dipole under the surfing of the magnetic field contour map, moved in a range of 0.11-0.12 m at $\mathrm{Z}$ direction, and the negative dipole moved in a distance about $0.106-0.116 \mathrm{~m}$ away from the measurement plane on the chest. In other words, the depth of two equivalent magnetic dipoles changed in a small region. Two moving magnetic dipoles are confined within the region of the heart. It is noticeable that $\mathrm{x}, \mathrm{y}, \mathrm{z}$ components curves of the location of two equivalent current dipoles are shown in Figure 9 and Figure 10. The first current dipole, i.e. the dominant dipole, moved in a range of 0.06-
$0.07 \mathrm{~m}$ at $\mathrm{Z}$ direction, and the accessorial equivalent dipole, moved in a distance of 0.11-0.14 $\mathrm{m}$ away from the measurement plane on the chest. In other words, the accessorial equivalent dipole located a little deeper than the dominant dipole. Two moving current dipoles are confined within the region of the heart.

In sum, two equivalent magnetic dipoles are moved in almost the same depth as the accessorial equivalent current dipole. The $\mathrm{x}$ and $\mathrm{y}$ components curves of the location of the accessorial equivalent current dipole have obvious sudden variation, however, two equivalent magnetic dipoles have no such phenomena.

\section{CONCLUSIONS}

Two multipole graphical models are introduced for the investigation of the cardiac electrical activity in this paper. Because the accuracy of graphical models depends on the accuracy of sources estimation, thus, the initial value

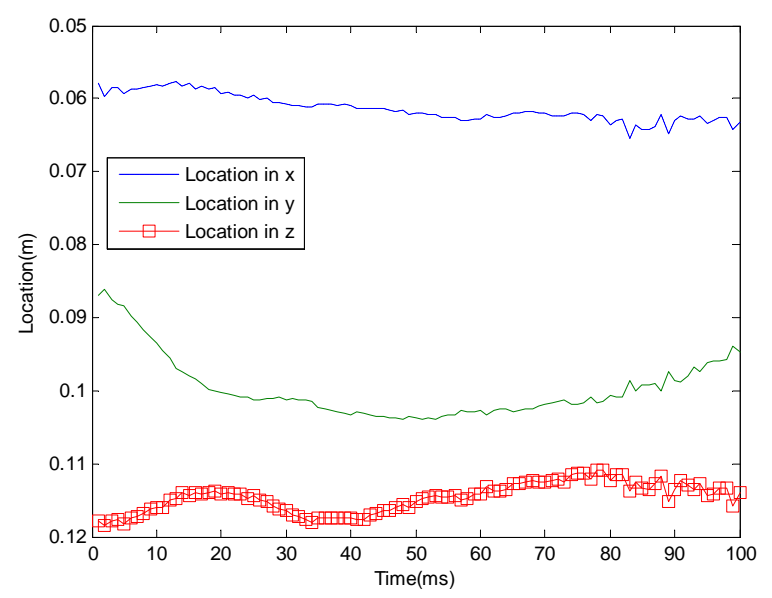

Figure 7. The time-varying location curves of the positive magnetic dipole.

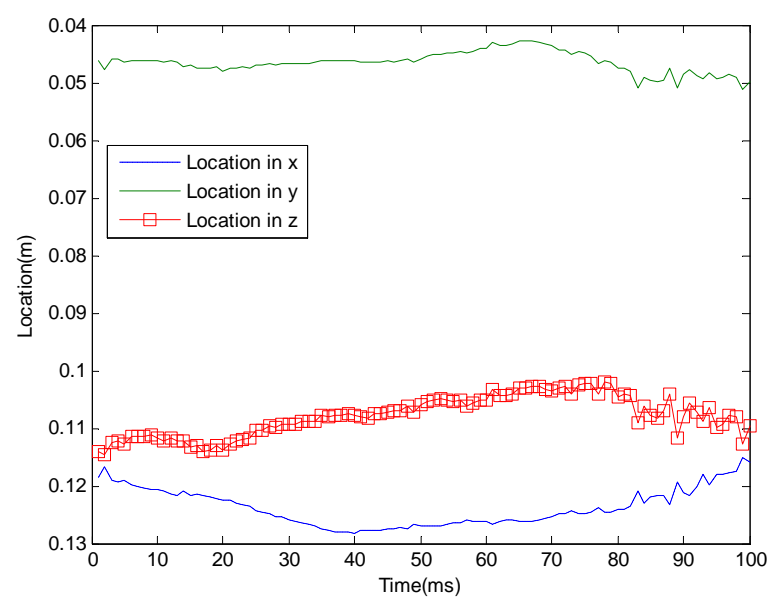

Figure 8. The time-varying location curves of the negative magnetic dipole. 


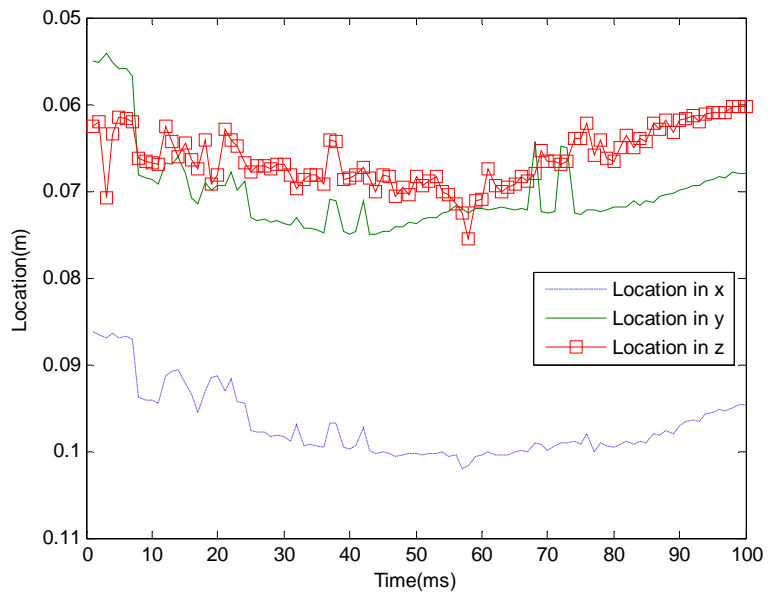

Figure 9. The time-varying location curves of the dominant current dipole.

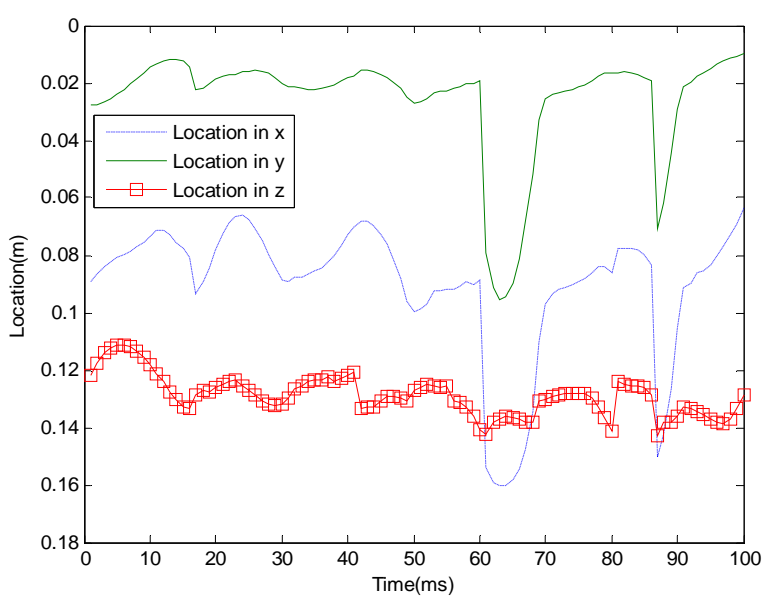

Figure 10. The time-varying location curves of the accessorial current dipole.

determining of source estimation is very important. The simulation results of the DCD model demonstrated that MFZL method mentioned above is an advisable approach to determine the initial values and the number of dipoles. Despite the simulation and the comparison results indicated that the both multipole graphical models have better GOF and RMSE, however, how to improve the accuracy of the graphical models still is a problem that remains to be solved. We have noted that two groups of reconstructed sources had some obvious difference on the accuracy of source localization, especially, the location variation of the accessorial current dipole in Figure 10. The time-varying location curves of the accessorial current dipole as shown in Figure $\mathbf{1 0}$ revealed some useful information of electrophysiological characteristics and the function information of the heart during ventricular repolarization, which needs analyzing together with the professional doctors in the future.

\section{ACKNOWLEDGMENTS}

This work obtained support from the National Natural Science Foundation of China (60771030), National High-Technology Research and Development Program of China (2008AA02Z308), the Shanghai Science and Technology Development Foundation (08JC 1421800), and the Open Project of State Key Laboratory of Function Materials for Information

\section{REFERENCES}

[1] D. Wu, H. C. Tsai, B. He. (1999) On the estimation of the Laplacian electrocardiogram during ventricular activation. Ann. Biomed. Eng., 27, 731-745.

[2] J. Haueisen, J. Schreiber, H. Brauer, and T. R. Knösche, (2002) Dependence of the inverse solution accuracy in magnetocardiography on the boundary-element discretization. IEEE Transactions On Magnetics, 38(2).

[3] S. Ohyu, Y. Okamoto, S. Kuriki. (2000) Use of the ventricular propagated excitation model in the magnetocar-diographic inverse problem for reconstruc tion of electrophysiological properties. IEEE Transactions on Biomedical Engineering, 49(6).

[4] S. Q. Jiang, L. Zhang, M. Chi, M. Luo, L. M. Wang. (2008) Dipole source localization by means of simplified double magnetic dipole model. International Journal of Bioelectromagnetism (IJBEM), 10(2).

[5] S. Jiang, M. Chi, L. Zhang, M. Luo, L. Wang. (2007) Dipole source localization in magnetocar diography. Proceedings of 2007 Joint Meeting of the 6th International Symposium on Noninvasive Functional Source Imaging of the Brain and Heart.

[6] M. Chi, S. Jiang, L. Zhang. (2008) Graphical model of cardiac electromagnetic source. International Conference on Bioinformatics and Biomedical Engineering (ICBBE' 08).

[7] S. Jiang, J. Dong, M. Chi, and W. Wang. (2008) A graphical model for the cardiac multi-dipole sources. Proceedings of the 5th International conference on Information Technology and Application in Biomedicine (ITAB'08), 434-436.

[8] S. Jiang, W. Y. Wang, J. M. Dong, and A. L. Li. (2008) modeling of bioelectrical activity by means of measured mcg data. Biomagetism-Transdisciplinary Research and Exploration, 241-243.

[9] W. Andra and H. Nowak. (1998) Magnetism in medicine. Germany, WILEY-VCH.

[10] B. He. (2004) Modeling and imaging of bioelectrical activity principles and applications. Kluwer Academic/ Plenum Publishers, 161-162. 